\title{
Serum BDNF levels and the antidepressant effects of electroconvulsive therapy with ketamine anaesthesia: a preliminary study
}

\author{
Wei Zheng ${ }^{\text {Equal first author, } 1}$, Qiaomei Cen Equal first author, ${ }^{1}$, Sha Nie ${ }^{1}$, Minyi Li ${ }^{1}$, Rong Zeng ${ }^{1}$, Sumiao Zhou ${ }^{1}$, Dong-Bin Cai ${ }^{2}$,

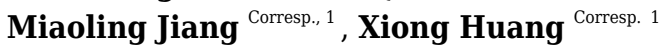 \\ ${ }^{1}$ the Affiliated Brain Hospital of Guangzhou Medical University, Guangzhou, China \\ 2 Shenzhen Traditional Chinese Medicine Hospital, Shenzhen, China \\ Corresponding Authors: Miaoling Jiang, Xiong Huang \\ Email address: miaoling2011_0318@163.com, 1195768576@qq.com
}

Objective: To first examine the relationship between serum brain-derived neurotrophic factor (BDNF) levels and antidepressant response to ketamine as an anaesthesia in electroconvulsive therapy (ECT) in Chinese patients with treatment-refractory depression (TRD). Methods: Thirty patients with TRD were enrolled and underwent eightECTsessions with ketamine $(0.8 \mathrm{mg} / \mathrm{kg})$ alone anaesthesia. Depression severity, response and remission were evaluated using the 17-item Hamilton Depression Rating Scale (HAMD-17). Enzymelinked immunosorbent assay (ELISA) was applied to examine serum BDNF levels in patients with TRD at baseline and after the second, fourth, and eighth ECT sessions. Baseline serumsampleswere also collectedfor 30 healthy controls. Results $\square$ No significant differences were observed in serum BDNF levels between patients with TRD and healthy controls at baseline ( $p>0.05)$. The remission rate was $76.7 \%(23 / 30)$ after the last ECT treatment, although all patients with TRD obtained antidepressant response criteria. Serum BDNF levels were not altered compared to baseline, even between remitters and nonremitters, despite the significant reduction in HAMD-17 and Brief Psychiatric Rating Scale (BPRS) scores after ECT with ketamine anaesthesia (all $p>0.05$ ). The antidepressant effects of ECT with ketamine anaesthesia were not correlated with changes in serum BDNF levels (all $p>0.05$ ). Conclusion: This preliminary study indicated that serum BDNF levels donot appear to be a reliable biomarker to determine the antidepressant effects of ketamine as an anaesthesia in ECT for patients with TRD. Further studies withlargersample sizes are warrantedtoconfirm these findings. 
Abstract: 240 words

Text: 2,246 words

Tables: 3

Figures: 2

Supplemental Tables: 1

Supplemental Figure: 2

\title{
Serum BDNF levels and the antidepressant effects of
}

\section{electroconvulsive therapy with ketamine anaesthesia: a preliminary}

\author{
study
}

\section{Running head: BDNF and ketamine}

1"Wei Zheng, MD, PhD;

1\#Qiao-Mei Cen, MD;

1 Sha Nie, MD;

1 Min-Yi Li, MD;

${ }^{1}$ Rong Zeng, MD;

1Su-Miao Zhou, MD;

2Dong-Bin Cai, MD;

$1 *$ Miao-Ling Jiang, MD;

$1 *$ Xiong Huang, MD;

1. The Affiliated Brain Hospital of Guangzhou Medical University (Guangzhou Huiai Hospital), Guangzhou, China;

2. Shenzhen Traditional Chinese Medicine Hospital, Shenzhen, China;

\#These authors contributed equally to this work.

*Address correspondence to Dr. Xiong Huang, the Affiliated Brain Hospital of Guangzhou Medical University (Guangzhou Huiai Hospital), Guangzhou, China; Fax: +86-20-81778484. Telephone: +86-18922165303. E-mail: 1195768576@qq.com; or Dr. Miao-Ling Jiang, the Affiliated Brain Hospital of Guangzhou Medical University (Guangzhou Huiai Hospital), Guangzhou, China; Fax: +86-20-81891425. Telephone: +86-18922165301. E-mail: miaoling2011_0318@163.com. 
38 Abstract

39 Objective: To first examine the relationship between serum brain-derived 40 neurotrophic factor (BDNF) levels and antidepressant response to ketamine 41 as an anaesthesia in electroconvulsive therapy (ECT) in Chinese patients 42 with treatment-refractory depression (TRD).

43 Methods: Thirty patients with TRD were enrolled and underwent 44 eight ECT sessions with ketamine $(0.8 \mathrm{mg} / \mathrm{kg})$ alone anaesthesia. 45 Depression severity, response and remission were evaluated using the 1746 item Hamilton Depression Rating Scale (HAMD-17). Enzyme-linked 47 immunosorbent assay (ELISA) was applied to examine serum BDNF levels in 48 patients with TRD at baseline and after the second, fourth, and eighth ECT 49 sessions. Baseline serum samples were also collected for 30 healthy controls.

Results: No significant differences were observed in serum BDNF levels

52 between patients with TRD and healthy controls at baseline $(p>0.05)$. The 53 remission rate was $76.7 \%(23 / 30)$ after the last ECT treatment, although all 54 patients with TRD obtained antidepressant response criteria. Serum BDNF 55 levels were not altered compared to baseline, even between remitters and 56 nonremitters, despite the significant reduction in HAMD-17 and Brief 57 Psychiatric Rating Scale (BPRS) scores after ECT with ketamine anaesthesia 58 (all $p>0.05$ ). The antidepressant effects of ECT with ketamine anaesthesia 
59 were not correlated with changes in serum BDNF levels (all $p>0.05$ ).

60 Conclusion: This preliminary study indicated that serum BDNF levels do not

61 appear to be a reliable biomarker to determine the antidepressant effects of

62 ketamine as an anaesthesia in ECT for patients with TRD. Further studies

63 with larger sample sizes are warranted to confirm these findings.

64 Keywords: Electroconvulsive therapy; ketamine; depression; brain-derived

65 neurotrophic factor 


\section{Introduction}

68 Electroconvulsive therapy (ECT) is widely considered to be the most effective

69 nonpharmacological therapy for mental disorders (Grover et al. 2018; Zong

70 et al. 2020), especially for major depressive disorder (MDD), despite

71 negative public perceptions (Dean \& Keshavan 2017; Gajaria \& Ravindran

72 2018; Sackeim et al. 2007). For example, Petrides et al. reported that the

73 remission rate was $87 \%$ for both psychotic and nonpsychotic patients with

74 MDD after an acute ECT course (Petrides et al. 2001). To minimize the

75 clinical risks and subjective unpleasantness during ECT, patients are

76 administered an intravenous anaesthetic, such as thiopental, methohexital,

77 propofol, ketamine or even a combination of ketamine and propofol (ketofol)

78 (Huang et al. 2020; Zheng et al. 2019a). A recent meta-analysis of 16 trials

$79(n=928)$ found that ketamine used in ECT accelerated the improvement of depressive symptoms in patients with MDD, with a short-term advantage in antidepressive effect at the early stages of ECT (Ren et al. 2018).

Ketamine, an N-methyl-d-aspartate receptor (NMDAR) antagonist, has been widely used as an analgesic, anaesthetic and antihyperalgesic agent (Radvansky et al. 2015). Interestingly, a single ketamine infusion at subanaesthesia doses elicited a rapid but time-limited antidepressant effect in treatment-refractory depression (TRD) (Hu et al. 2016; Zarate et al. 2006). Repeated ketamine infusions at sub-anaesthesia doses have a cumulative 
and sustained antidepressant effect on TRD (Phillips et al. 2019; Zheng et al. 2018; Zheng et al. 2019b). Thus, ketamine used as an anaesthesia in ECT may enhance the antidepressant effects of ECT, while also having rapid independent antidepressant properties itself (Erdil et al. 2015; Kranaster et al. 2011; Okamoto et al. 2010; Ren et al. 2018; Zheng et al. 2019a). Zhong et al. found that ketamine anaesthesia achieved earlier antidepressant efficacy and a higher rate of remission than propofol anaesthesia and ketofol anaesthesia in TRD treated with ECT, suggesting that ketamine alone in ECT may represent an optimized therapy for TRD (Zhong et al. 2016).

When compared to healthy controls, patients with TRD have low serum BDNF levels (Molendijk et al. 2014; Polyakova et al. 2015b). Many, but not all studies (Brunoni et al. 2008; Groves 2007; Polyakova et al. 2015b) found that low serum BDNF levels in patients with TRD were normalized after obtaining an antidepressant response. A few studies have examined the correlation of blood BDNF levels and the antidepressant response to ECT with ketamine anaesthesia or ketofol anaesthesia, but with inconsistent findings. For instance, a recent study reported that ECT treatment with ketamine anaesthesia, but not with methohexital anaesthesia, significantly increased plasma BDNF levels (Carspecken et al. 2018). However, another study found that ECT with ketofol anaesthesia was not correlated with enhanced serum BDNF levels (Huang et al. 2020). Moreover, baseline serum 
109 BDNF levels did not appear to be an eligible biomarker for predicting the

110 antidepressant response to ECT with ketofol anaesthesia, as measured using

111 the 17-item Hamilton Depression Rating Scale (HAMD-17) (Huang et al. 112 2020).

113 To date, no study has been conducted to examine whether serum BDNF 114 levels predict a rapid antidepressant response to ketamine used alone as an 115 anaesthesia during ECT in Chinese patients with TRD. In this study, thirty 116 Chinese patients with TRD were administered ketamine alone as an 117 anaesthesia in ECT. We hypothesized that (1) patients with TRD would 118 exhibit lower serum BDNF levels than healthy controls and (2) the 119 antidepressant response to ketamine anaesthesia in ECT would be correlated 120 with serum BDNF levels.

\section{Methods}

123 The protocol for this study was approved by the ethics review board of the 124 Affiliated Brain Hospital of Guangzhou Medical University (Ethics Number: 125 [2013]020). This study was conducted following the Declaration of Helsinki 126 and was performed between February 2013 and December 2013.

\section{Participants}


129 All patients were recruited from the wards of the Affiliated Brain Hospital of 130 Guangzhou Medical University based on the following criteria: (1) age from 13118 to 65 years; (2) diagnosed with major depression according to the 132 International Statistical Classification of Diseases and Related Health 133 Problems, 10th revision (ICD-10) with a current major depressive episode; 134 (3) having severe depressive symptoms (HAMD-17 scores $\geq 20$ ) at screening; 135 (4) did not respond adequately to appropriate courses of at least two antidepressants in the current episode (Huang et al. 2020; Zheng et al. 137 2020); (5) had no a history of severe physical illness (i.e., Parkinson disease) 138 and no drug or alcohol abuse; (6) had no a history of seizures; (7) was not breastfeeding or pregnant; and (8) had no any contraindication for ketamine anaesthesia and ECT. All patients with TRD provided written informed 141 consent at the beginning of participation.

142 Thirty age and sex-matched apparently healthy volunteers were 143 recruited from the local community during the same phase with no alcohol or 144 other substance abuse/dependence or serious physical diseases. All healthy 145 volunteers provided written informed consent at the beginning of 146 participation. 
149 All patients with TRD received eight ECT treatments with ketamine 150 anaesthesia alone $(0.8 \mathrm{mg} / \mathrm{kg})$. ECT treatment was performed three times 151 per week for three consecutive weeks for a total of eight treatments. During 152 the courses of ECT, no psychiatric medications were prescribed to the 153 subjects. The seizure threshold of each patient was determined based on the 154 half-age method (\% energy=half the age) (Petrides \& Fink 1996; Yasuda et 155 al. 2015), and bitemporal ECT for each case was conducted by using 156 Thymatron ${ }^{\circledR}$ IV device (Somatics LLC, Lake Bluff, Illinois, USA). Vital signs, 157 such as temperature and heart rate, were regularly recorded.

\section{Clinical Assessment}

The HAMD-17 (Hamilton 1960) and the Brief Psychiatric Rating Scale (BPRS)

161 were used to assess the severity of depressive and psychotic symptoms, 162 respectively, at baseline, after the second, fourth, and eighth sessions of 163 ECT. Antidepressant response was defined as a $50 \%$ or greater reduction in 164 HAMD-17 scores (Lin \& Lin 2019) and antidepressant remission as HAMD-17 165 scores $\leq 6$ (Riedel et al. 2010). Riedel et al. found that a HAMD-17 cut-of $\leq 6$ 166 (area under the curve $=0.90$ ) was correlated with a maximum sensitivity and 167 specificity for defining remission criteria (Riedel et al. 2010). 
170 Blood sample were collected from healthy controls at baseline. Blood

171 samples from patients with TRD were collected at baseline and after the

172 second, fourth, and eighth treatments of ECT. Before analysis of serum

173 BDNF levels, blood samples were stored at $-80^{\circ} \mathrm{C}$. In line with the

174 manufacturer's instructions, an enzyme-linked immunosorbent assay (ELISA)

175 kit (BDNF Emax Immunoassay System, Promega, USA) was used to examine

176 serum BDNF levels. Absorbance at $450 \mathrm{~nm}$ wavelength was used to analyse

177 BDNF concentrations based on a standard curve.

178

179 Data analyses

180 Comparison of demographics and clinical characteristics between healthy 181 controls and patients with TRD, and between remitters and nonremitters 182 defined as HAMD-17 scores $\leq 6$ (Riedel et al. 2010), was examined using the 183 Student's t-test, Mann-Whitney U-test, chi-square test, or Fisher's exact test, 184 as appropriate. A linear mixed model was used to compare serum BDNF 185 levels and the severity of depressive and psychotic symptoms between 186 remitters and nonremitters following eight ECT treatments. The correlation 187 between changes in illness severity and changes in serum BDNF levels was 188 analysed using Pearson's bivariate correlation analysis. All outcomes were 189 analysed using IBM SPSS statistics version 25.0 , and $p<0.05$ was considered 190 significant. 


\section{Results}

\section{Participant characteristics}

194

195

196

197

198

199

200

201

202

203

204

205

206

207

208

209

210

211

As shown in Table 1 and Supplemental Figure 1, no significant differences were observed in baseline serum BDNF levels between healthy controls and patients with TRD $(p>0.05)$. Similarly, no significant differences in terms of baseline serum BDNF levels, HAMD-17 scores or BPRS scores were found between remitters and nonremitters (all $p>0.05$, Table 2 ).

\section{Treatment remission and serum BDNF levels}

After the last ECT treatment, the remission rate was $76.7 \%(23 / 30)$, while the response rate was $100 \%(30 / 30)$ (Table 1$)$. Significant reduction in illness severity as measured by HAMD-17 (Figure 1) and BPRS (Supplemental Figure 2) was observed following eight ECT treatments. No significant difference in serum BDNF levels was found at any of the indicated times between remitters and nonremitters, even when compared to baseline across the total sample (all $p>0.05$, Figure 2 ). In the linear mixed model, serum BDNF levels showed no significant main effects for group, time, or group-by-time interactions (all p>0.05, Table 3 ). Changes in HAMD-17 and BPRS scores following ECT treatment between remitters and nonremitters was also analysed using a linear mixed model (Table 3 ). 


\section{Association between serum BDNF levels and illness severity}

214 As reported in Supplemental Table 1, there were no significant correlations

215 between serum BDNF levels and illness severity as measured by HAMD-17

216 and BPRS.

217

218

Discussion

219

This study was, to our knowledge, the first to examine the relationship

of serum BDNF levels and the rapid antidepressant effects of ECT with

ketamine anaesthesia in Chinese patients with TRD. The main findings of the

current study included the following: 1 ) there was no significant difference in baseline serum BDNF levels between healthy controls and patients with TRD;

2) following eight sessions of ECT with ketamine anaesthesia, all patients with TRD met the response criteria, and $76.7 \%$ remitted based on the criteria reported by Riedel et al.'s study (Riedel et al. 2010); 3) ketamine used in ECT did not alter serum BDNF levels compared to baseline, and serum BDNF levels between remitters and nonremitters was not significantly different; 4) there was no significant correlation between serum BDNF levels and improvement of depressive symptoms following eight sessions of ECT with ketamine anaesthesia. 
233 al. 2020; Maffioletti et al. 2019; Polyakova et al. 2015a; Ryan et al. 2018), 234 no significant difference was observed in serum BDNF levels between 235 patients with TRD and healthy controls. However, other studies have 236 reported that patients with depression exhibit reduced serum BDNF levels 237 compared to healthy controls (Allen et al. 2015; Karege et al. 2002; Kishi et 238 al. 2017; Matrisciano et al. 2009; Nase et al. 2016; Rapinesi et al. 2015; 239 Wolkowitz et al. 2011; Zheng et al. 2020), suggesting that serum BDNF 240 deficit might represent a potential biomarker in patients with TRD. However, 241 low serum BDNF levels were not specific to patients with TRD, but were also 242 observed in patients with schizophrenia and mania, and even in patients 243 with acne vulgaris (Karamustafalioglu et al. 2015; Li et al. 2016; Mikhael et 244 al. 2019; Mora et al. 2019). Taken together, blood BNDF level appears to 245 have no value for diagnosis of depression in clinical practice.

After completing eight ECT treatments with ketamine as an aesthesia, $247100 \%$ of patients with TRD responded in this study, and $76.7 \%$ met 248 remission criteria, similar with the prior findings (Huang et al. 2020). Huang 249 et al. found that thirty patients with TRD underwent eight ECT treatments 250 with ketofol as aesthesia where $100 \%$ responded and $53.3 \%$ remitted 251 (Huang et al. 2020). Zhong et al. reported higher remission rates for 252 patients with TRD receiving ECT with ketamine alone than propofol alone 253 and ketofol, indicating that ketamine used alone as an aesthesia in ECT may 
254 be considered an optimal treatment in Chinese patients with TRD (Zhong et

al. 2016). Importantly, the findings of the current study also support the 256 results of a meta-analysis of randomized controlled trials that ketamine used 257 in ECT accelerates the antidepressive response and remission in depressed 258 patients (Ren et al. 2018).

259 Consistent with findings of previous studies (Allen et al. 2015; Huang et 260 al. 2020), serum BDNF levels did not significantly change during ECT with 261 ketamine anaesthesia in this study. For example, Huang et al. found that 262 ECT with ketofol anaesthesia did not alter serum BDNF levels, despite its 263 rapidly decreasing depressive symptoms (Huang et al. 2020). However, 264 findings on the effect of ECT with other anaesthesia types, such as 265 methohexital or thiopental sodium, on serum BDNF levels are inconsistent 266 (Maffioletti et al. 2019; Vanicek et al. 2019b). For example, Vanicek et al. 267 found a significant increase in serum BDNF levels after continuation ECT 268 treatments with methohexital (Vanicek et al. 2019b), while another study 269 found that ECT with thiopental sodium did not alter serum BDNF levels 270 (Maffioletti et al. 2019).

271 The current study also confirmed the findings of previous studies that 272 the antidepressant effects of ECT with ketamine anaesthesia were not 273 correlated with serum BDNF levels (Allen et al. 2015; Huang et al. 2020; 274 Kishi et al. 2017). Similar to nonconvulsive electrotherapy (NET) using 
275 standard ECT technique but below seizure threshold, the change in serum

276 BDNF levels in patients with TRD was not correlated with the antidepressant

277 effects of NET (Zheng et al. 2020). A recent study concluded that serum

278 BDNF levels in patients with late-life unipolar depression cannot be

279 considered an eligible biomarker for the antidepressant effects of ECT (van

280 Zutphen et al. 2019).

281 It is important to mention the following limitations of this study. The 282 primary limitation of this study is the small sample size, partly accounting for 283 the negative findings. Another limitation of the current study was the open284 label design, limiting the interpretation of efficacy. For instance, Carspecken 285 et al. found that ketamine used in ECT does not significantly improve 286 depressive symptoms compared to methohexital used in ECT (Carspecken et 287 al. 2018). Finally, the absence of follow-up visits after the entire treatment 288 for this study limited our capacity to further examine how long the 289 antidepressant effects of ECT with ketamine persists and whether increased 290 BDNF concentrations require a longer time to manifest. For instance, Vanicek 291 et al. found that peak serum BDNF levels were achieved one month after the 292 final ECT treatment (Vanicek et al. 2019a).

293 In conclusion, this preliminary study indicated that serum BDNF levels 294 do not appear to be a reliable biomarker to determine the antidepressant 


\section{Acknowledgements}

299

300

301

302

303

304

305

306

307

308

309

310

311

312

313

314

315

316

317

318

319

320

321

322

This study was funded by the Science and Technology Planning Project of Guangdong Province (B2016109), Science and Technology Planning Project of Liwan District of Guangzhou (202004034), and Guangzhou Clinical Characteristic Technology Project (2019TS67).

\section{Conflict of Interest}

The authors have no conflicts of interest concerning this article.

\section{References}

Allen AP, Naughton M, Dowling J, Walsh A, Ismail F, Shorten G, Scott L, McLoughlin DM, Cryan JF, Dinan TG, Clarke G. 2015. Serum BDNF as a peripheral biomarker of treatment-resistant depression and the rapid antidepressant response: A comparison of ketamine and ECT. J Affect Disord 186:306-311.

Brunoni AR, Lopes M, and Fregni F. 2008. A systematic review and meta-analysis of clinical studies on major depression and BDNF levels: implications for the role of neuroplasticity in depression. Int J Neuropsychopharmacol 11:1169-1180.

Carspecken CW, Borisovskaya A, Lan ST, Heller K, Buchholz J, Ruskin D, and Rozet I. 2018. Ketamine anesthesia does not improve depression scores in electroconvulsive therapy: a randomized clinical trial. J Neurosurg Anesthesiol 30:305-313.

Dean J, and Keshavan M. 2017. The neurobiology of depression: An integrated view. Asian J Psychiatr 27:101-111.

Erdil F, Ozgul U, Çolak C, Cumurcu B, and Durmus M. 2015. Effect of the addition of ketamine to sevoflurane anesthesia on seizure duration in electroconvulsive therapy. J ect 31:182-185.

Fernandes B, Gama CS, Massuda R, Torres M, Camargo D, Kunz M, Belmonte-de-Abreu PS, Kapczinski F, de Almeida Fleck MP, and Inês Lobato M. 2009. Serum brain-derived neurotrophic factor (BDNF) is not associated with response to electroconvulsive therapy $(\mathrm{ECT})$ : a pilot study in drug resistant depressed patients. 
Neurosci Lett 453:195-198.

Gajaria A, and Ravindran AV. 2018. Interventions for perinatal depression in low and middle-income countries: A systematic review. Asian J Psychiatr 37:112-120.

Grover S, Satapathy A, Chakrabarti S, and Avasthi A. 2018. Electroconvulsive therapy among elderly patients: a study from tertiary care centre in north India. Asian J Psychiatr 31:43-48.

Groves JO. 2007. Is it time to reassess the BDNF hypothesis of depression? Mol Psychiatry 12:1079-1088.

Hamilton M. 1960. A rating scale for depression. J Neurol Neurosurg Psychiatry 23:56-62.

Hu YD, Xiang YT, Fang JX, Zu S, Sha S, Shi H, Ungvari GS, Correll CU, Chiu HF, Xue Y, Tian TF, Wu AS, Ma X, Wang G. 2016. Single i.v. ketamine augmentation of newly initiated escitalopram for major depression: results from a randomized, placebo-controlled 4-week study. Psychol Med 46:623-635.

Huang XB, Huang X, He HB, Mei F, Sun B, Zhou SM, Yan S, Zheng W, and Ning YP. 2020. BDNF and the antidepressant effects of ketamine and propofol in electroconvulsive therapy: a preliminary study. Neuropsychiatr Dis Treat 16:901-908.

Karamustafalioglu N, Genc A, Kalelioglu T, Tasdemir A, Umut G, Incir S, Akkuş M, and Emul M. 2015. Plasma BDNFs level initially and post treatment in acute mania: comparison between ECT and atypical antipsychotic treatment and healthy controls. J Psychopharmacol 29:898-902.

Karege F, Perret G, Bondolfi G, Schwald M, Bertschy G, and Aubry JM. 2002. Decreased serum brain-derived neurotrophic factor levels in major depressed patients. Psychiatry Res 109:143-148.

Kishi T, Yoshimura R, Ikuta T, and Iwata N. 2017. Brain-derived neurotrophic factor and major depressive disorder: evidence from meta-analyses. Front Psychiatry 8:308.

Kranaster L, Kammerer-Ciernioch J, Hoyer C, and Sartorius A. 2011. Clinically favourable effects of ketamine as an anaesthetic for electroconvulsive therapy: a retrospective study. Eur Arch Psychiatry Clin Neurosci 261:575-582.

Li J, Ye F, Xiao W, Tang X, Sha W, Zhang X, and Wang J. 2016. Increased serum brain-derived neurotrophic factor levels following electroconvulsive therapy or antipsychotic treatment in patients with schizophrenia. Eur Psychiatry 36:23-28.

Lin HS, and Lin CH. 2019. Early improvement in HAMD-17 and HAMD-6 scores predicts ultimate response and remission for depressed patients treated with fluoxetine or ECT. J Affect Disord 245:91-97.

Maffioletti E, Gennarelli M, Gainelli G, Bocchio-Chiavetto L, Bortolomasi M, and Minelli A. 2019. BDNF genotype and baseline serum levels in relation to electroconvulsive therapy effectiveness in treatment-resistant depressed patients. J ECT 35:189-194.

Matrisciano F, Bonaccorso S, Ricciardi A, Scaccianoce S, Panaccione I, Wang L, Ruberto A, Tatarelli R, Nicoletti F, Girardi P, Shelton R. 2009. Changes in BDNF serum levels in patients with major depression disorder (MDD) after 6 months treatment with sertraline, escitalopram, or venlafaxine. J Psychiatr Res 43:247-254.

Mikhael NW, Hamed AM, Mansour AI, and Abdelrahman ES. 2019. Serum levels of brain-derived neurotrophic factor in patients with acne vulgaris. J Cosmet Dermatol 18:1998-2003.

Molendijk ML, Spinhoven P, Polak M, Bus BA, Penninx BW, and Elzinga BM. 2014. Serum BDNF concentrations as peripheral manifestations of depression: evidence from a systematic review and meta-analyses on 179 associations (N=9484). Mol Psychiatry 19:791-800.

Mora E, Portella MJ, Piñol-Ripoll G, López R, Cuadras D, Forcada I, Teres M, Vieta E, and Mur M. 2019. High BDNF serum levels are associated to good cognitive functioning in bipolar disorder. Eur Psychiatry 60:97-107. 
Nase S, Köhler S, Jennebach J, Eckert A, Schweinfurth N, Gallinat J, Lang UE, and Kühn S. 2016. Role of serum brain derived neurotrophic factor and central n-acetylaspartate for clinical response under antidepressive pharmacotherapy. Neurosignals 24:1-14.

Okamoto N, Nakai T, Sakamoto K, Nagafusa Y, Higuchi T, and Nishikawa T. 2010. Rapid antidepressant effect of ketamine anesthesia during electroconvulsive therapy of treatment-resistant depression: comparing ketamine and propofol anesthesia. J ECT 26:223-227.

Petrides G, and Fink M. 1996. The "half-age" stimulation strategy for ECT dosing. Convuls Ther 12:138-146.

Petrides G, Fink M, Husain MM, Knapp RG, Rush AJ, Mueller M, Rummans TA, O'Connor KM, Rasmussen KG, Jr, Bernstein HJ, Biggs M, Bailine S H, Kellner C H. 2001. ECT remission rates in psychotic versus nonpsychotic depressed patients: a report from CORE. J ECT 17:244-253.

Phillips JL, Norris S, Talbot J, Birmingham M, Hatchard T, Ortiz A, Owoeye O, Batten LA, and Blier P. 2019. Single, repeated, and maintenance ketamine infusions for treatment-resistant depression: a randomized controlled trial. Am J Psychiatry 176:401-409.

Polyakova M, Schroeter ML, Elzinga BM, Holiga S, Schoenknecht P, de Kloet ER, and Molendijk ML. 2015a. Brainderived neurotrophic factor and antidepressive effect of electroconvulsive therapy: systematic review and meta-analyses of the preclinical and clinical literature. PLoS One 10:e0141564.

Polyakova M, Stuke K, Schuemberg K, Mueller K, Schoenknecht P, and Schroeter ML. 2015b. BDNF as a biomarker for successful treatment of mood disorders: a systematic \& quantitative meta-analysis. J Affect Disord 174:432-440.

Radvansky BM, Shah K, Parikh A, Sifonios AN, Le V, and Eloy JD. 2015. Role of ketamine in acute postoperative pain management: a narrative review. Biomed Res Int 2015:749837.

Rapinesi C, Kotzalidis GD, Curto M, Serata D, Ferri VR, Scatena P, Carbonetti P, Napoletano F, Miele J, Scaccianoce S, Del Casale A, Nicoletti F, Angeletti G, Girardi P. 2015. Electroconvulsive therapy improves clinical manifestations of treatment-resistant depression without changing serum BDNF levels. Psychiatry Res 227:171-178.

Ren L, Deng J, Min S, Peng L, and Chen Q. 2018. Ketamine in electroconvulsive therapy for depressive disorder: A systematic review and meta-analysis. J Psychiatr Res 104:144-156.

Riedel M, Möller HJ, Obermeier M, Schennach-Wolff R, Bauer M, Adli M, Kronmüller K, Nickel T, Brieger P, Laux G , Bender W, Heuser I, Zeiler J, Gaebel W, Seemüller F. 2010. Response and remission criteria in major depression--a validation of current practice. J Psychiatr Res 44:1063-1068.

Ryan KM, Dunne R, and McLoughlin DM. 2018. BDNF plasma levels and genotype in depression and the response to electroconvulsive therapy. Brain Stimul 11:1123-1131.

Sackeim HA, Prudic J, Fuller R, Keilp J, Lavori PW, and Olfson M. 2007. The cognitive effects of electroconvulsive therapy in community settings. Neuropsychopharmacology 32:244-254.

van Zutphen EM, Rhebergen D, van Exel E, Oudega ML, Bouckaert F, Sienaert P, Vandenbulcke M, Stek M, and Dols A. 2019. Brain-derived neurotrophic factor as a possible predictor of electroconvulsive therapy outcome. Transl Psychiatry 9:155.

Vanicek T, Kranz GS, Vyssoki B, Fugger G, Komorowski A, Höflich A, Saumer G, Milovic S, Lanzenberger R, Eckert A, Kasper S, Frey R. 2019a. Acute and subsequent continuation electroconvulsive therapy elevates serum BDNF levels in patients with major depression. Brain Stimul 12:1041-1050.

Vanicek T, Kranz GS, Vyssoki B, Komorowski A, Fugger G, Höflich A, Micskei Z, Milovic S, Lanzenberger R, Eckert 
405

406

407

408

409

410

411

412

413

414

415

416

417

418

419

420

421

422

423

424

425

426

427

428

429

430

431

432

433

434
A, Kasper S, Frey R. 2019b. Repetitive enhancement of serum BDNF subsequent to continuation ECT. Acta Psychiatr Scand 140:426-434.

Wolkowitz OM, Wolf J, Shelly W, Rosser R, Burke HM, Lerner GK, Reus VI, Nelson JC, Epel ES, and Mellon SH. 2011. Serum BDNF levels before treatment predict SSRI response in depression. Prog Neuropsychopharmacol Biol Psychiatry 35:1623-1630.

Yasuda K, Kobayashi K, Yamaguchi M, Tanaka K, Fujii T, Kitahara Y, Tamaoki T, Matsushita Y, Nunomura A, and Motohashi N. 2015. Seizure threshold and the half-age method in bilateral electroconvulsive therapy in Japanese patients. Psychiatry Clin Neurosci 69:49-54.

Zarate CA, Jr., Singh JB, Carlson PJ, Brutsche NE, Ameli R, Luckenbaugh DA, Charney DS, and Manji HK. 2006. A randomized trial of an $\mathrm{N}$-methyl-D-aspartate antagonist in treatment-resistant major depression. Arch Gen Psychiatry 63:856-864.

Zheng W, Jiang ML, He HB, Li RP, Li QL, Zhang CP, Zhou SM, Yan S, Ning YP, and Huang X. 2020. Serum BDNF levels are not associated with the antidepressant effects of nonconvulsive electrotherapy. Neuropsychiatr Dis Treat 16:1555-1560.

Zheng W, Li XH, Zhu XM, Cai DB, Yang XH, Ungvari GS, Ng CH, Ning YP, Hu YD, He SH, Wang G, Xiang YT. 2019a. Adjunctive ketamine and electroconvulsive therapy for major depressive disorder: A meta-analysis of randomized controlled trials. J Affect Disord 250:123-131.

Zheng W, Zhou YL, Liu WJ, Wang CY, Zhan YN, Li HQ, Chen LJ, Li MD, and Ning YP. 2018. Rapid and longer-term antidepressant effects of repeated-dose intravenous ketamine for patients with unipolar and bipolar depression. J Psychiatr Res 106:61-68.

Zheng W, Zhou YL, Liu WJ, Wang CY, Zhan YN, Li HQ, Chen L, Li MD, and Ning YP. 2019b. Investigation of medical effect of multiple ketamine infusions on patients with major depressive disorder. J Psychopharmacol 33:494-501.

Zhong X, He H, Zhang C, Wang Z, Jiang M, Li Q, Zhang M, and Huang X. 2016. Mood and neuropsychological effects of different doses of ketamine in electroconvulsive therapy for treatment-resistant depression. J Affect Disord 201:124-130.

Zong QQ, Qi H, Wang YY, Zhang C, Balbuena L, Ungvari GS, An FR, and Xiang YT. 2020. Knowledge and attitudes of adolescents with psychiatric disorders and their caregivers towards electroconvulsive therapy in China. Asian J Psychiatr 49:101968. 


\section{Table $\mathbf{1}$ (on next page)}

Comparison of demographic and clinical characteristics between patients with TRD and healthy controls

${ }^{\mathrm{a} A n t i d e p r e s s a n t ~ r e s p o n s e ~ w a s ~ d e f i n e d ~ a s ~ a ~} 50 \%$ or greater reduction in HAMD-17 scores.

${ }^{b}$ Antidepressant remission was defined as HAMD-17 scores $\leq 6$ as recommended by Riedel et al.'s study. Abbreviations: BDNF=brain-derived neurotrophic factor; $d f=$ degrees of freedom; $\mathrm{ECT}=$ electroconvulsive therapy; $\mathrm{SD}=$ standard deviation; $\mathrm{TRD}=$ treatment-refractory depression. 
1 Table 1. Comparison of demographic and clinical characteristics between patients with 2 TRD and healthy controls

3

\begin{tabular}{|l|c|c|c|c|c|c|c|}
\hline \multirow{2}{*}{ Variables } & \multicolumn{2}{|c|}{$\begin{array}{c}\text { Patients with TRD } \\
(\mathrm{n}=30)\end{array}$} & $\begin{array}{c}\text { Healthy controls } \\
(\mathrm{n}=30)\end{array}$ & \multicolumn{3}{c|}{ Statistics } \\
\cline { 2 - 8 } & $\mathrm{n}$ & $\%$ & $\mathrm{n}$ & $\%$ & $\mathrm{X}^{2}$ & $\mathrm{df}$ & $P$ \\
\hline Male & 14 & 46.7 & 13 & 43.3 & 0.07 & 1 & 0.795 \\
\hline Employed & 24 & 80.0 & - & - & - & - & - \\
\hline Married & 20 & 66.7 & - & - & - & - & - \\
\hline $\begin{array}{l}\text { Antidepressant } \\
\text { response after } \\
\text { the last ECT }\end{array}$ & 30 & 100.0 & - & - & - & - & - \\
\hline $\begin{array}{l}\text { Antidepressant } \\
\text { remission after } \\
\text { the last ECT }\end{array}$ & 23 & 76.7 & - & - & - & - & - \\
\hline & Mean & SD & Mean & SD & $t$ & df & $P$ \\
\hline Age (years) & 32.1 & 9.9 & 30.8 & 8.2 & 0.58 & 58 & 0.561 \\
\hline $\begin{array}{l}\text { Baseline serum } \\
\text { BDNF levels } \\
\text { (ng/ml) }\end{array}$ & 23.7 & 8.9 & 24.2 & 6.1 & -0.24 & 58 & 0.810 \\
\hline
\end{tabular}

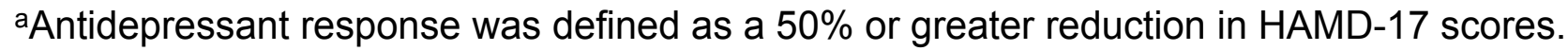

${ }^{b}$ Antidepressant remission was defined as HAMD-17 scores $\leq 6$ as recommended by Riedel et al.'s study.

Abbreviations: $B D N F=$ brain-derived neurotrophic factor; $d f=$ degrees of freedom; $\mathrm{ECT}=$ =electroconvulsive therapy; $\mathrm{SD}=$ standard deviation; $\mathrm{TRD}=$ treatment-refractory depression. 


\section{Table 2 (on next page)}

Comparison of demographic and clinical characteristics between remitters and nonremitters after ECT

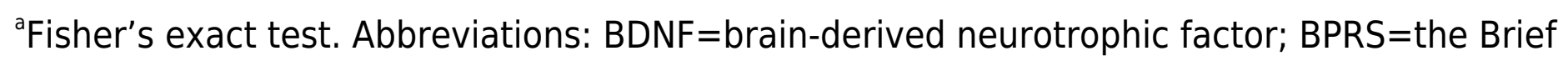

Psychiatric Rating Scale; $d f=$ degrees of freedom; ECT=electroconvulsive therapy;

HAMD-17=the 17-item Hamilton Depression Rating Scale ; SD=standard deviation. 
1 Table 2. Comparison of demographic and clinical characteristics between remitters and 2 nonremitters after ECT

\begin{tabular}{|c|c|c|c|c|c|c|c|c|c|}
\hline \multirow[t]{2}{*}{ Variables } & \multicolumn{2}{|c|}{$\begin{array}{l}\text { Total samples } \\
\qquad(n=30)\end{array}$} & \multicolumn{2}{|c|}{$\begin{array}{l}\text { Remitters } \\
\quad(n=23)\end{array}$} & \multicolumn{2}{|c|}{$\begin{array}{l}\text { Nonremitters } \\
\qquad(\mathrm{n}=7)\end{array}$} & \multicolumn{3}{|c|}{ Statistics } \\
\hline & $\mathrm{n}$ & $\%$ & $\mathrm{n}$ & $\%$ & $\mathrm{n}$ & $\%$ & $x^{2}$ & df & $P$ \\
\hline Male & 14 & 46.6 & 10 & 43.4 & 4 & 57.1 & 0.4 & 1 & 0.526 \\
\hline Employed & 24 & 80.0 & 19 & 82.6 & 5 & 71.4 & $--^{-a}$ & $---^{a}$ & 0.603 \\
\hline Married & 20 & 66.7 & 14 & 60.8 & 6 & 85.7 & $--^{a}$ & $---a$ & 0.372 \\
\hline & Mean & SD & Mean & SD & Mean & SD & $t / z$ & df & $P$ \\
\hline Age (years) & 32.1 & 9.9 & 31.2 & 10.5 & 35.3 & 7.5 & 0.9 & 28 & 0.344 \\
\hline $\begin{array}{l}\text { Baseline serum } \\
\text { BDNF levels } \\
\text { (ng/ml) }\end{array}$ & 23.7 & 8.9 & 24.3 & 9.8 & 21.8 & 5.8 & -0.6 & 28 & 0.543 \\
\hline $\begin{array}{l}\text { Baseline HAMD- } \\
17 \text { scores }\end{array}$ & 26.7 & 1.6 & 26.7 & 1.7 & 26.8 & 1.1 & 0.3 & 28 & 0.769 \\
\hline $\begin{array}{l}\text { Baseline BPRS } \\
\text { scores }\end{array}$ & 35.5 & 4.2 & 35.6 & 4.5 & 35.0 & 3.1 & -0.3 & 28 & 0.741 \\
\hline
\end{tabular}




\section{Table 3 (on next page)}

Comparison of serum BDNF levels, HAMD-17 scores, and BPRS scores between remitters and nonremitters using linear mixed model analysis

Bolded values are $p<0.05$. Abbreviations: $B D N F=$ brain-derived neurotrophic factor; BPRS=the Brief Psychiatric Rating Scale; HAMD-17=the 17-item Hamilton Depression Rating Scale. 
1 Table 3. Comparison of serum BDNF levels, HAMD-17 scores, and BPRS scores

2 between remitters and nonremitters using linear mixed model analysis

3

\begin{tabular}{|l|c|c|c|c|c|c|}
\hline \multirow{2}{*}{ Variables } & \multicolumn{2}{|c|}{$\begin{array}{c}\text { Group-by-time } \\
\text { interaction }\end{array}$} & \multicolumn{2}{c|}{ Time main effect } & \multicolumn{2}{c|}{ Group main effect } \\
\cline { 2 - 7 } & $F$ & $P$ & $F$ & $P$ & $F$ & $P$ \\
\hline Serum BDNF levels & 1.2 & 0.321 & 1.6 & 0.214 & 0.9 & 0.355 \\
\hline HAMD-17 scores & 3.7 & $\mathbf{0 . 0 1 9}$ & 2095.5 & $<\mathbf{0 . 0 0 1}$ & 5.0 & $\mathbf{0 . 0 2 8}$ \\
\hline BPRS scores & 1.2 & 0.309 & 332.1 & $<\mathbf{0 . 0 0 1}$ & 1.6 & 0.208 \\
\hline
\end{tabular}

Bolded values are $p<0.05$.

Abbreviations: $B D N F=$ brain-derived neurotrophic factor; BPRS=the Brief Psychiatric Rating Scale; HAMD-17=the 17-item Hamilton Depression Rating Scale. 


\section{Figure 1}

Changes in depressive symptoms following eight ECT treatments

*Significant difference was found at any of the indicated times when compared baseline $(p<0.05) .{ }^{~ " S i g n i f i c a n t ~ d i f f e r e n c e ~ w a s ~ f o u n d ~ b e t w e e n ~ r e m i t t e r s ~ a n d ~ n o n r e m i t t e r s ~ a t ~ i n d i c a t e d ~}$ times $(p<0.05)$. Abbreviations: ECT=electroconvulsive therapy; HAMD-17=the 17 -item Hamilton Depression Rating Scale . 


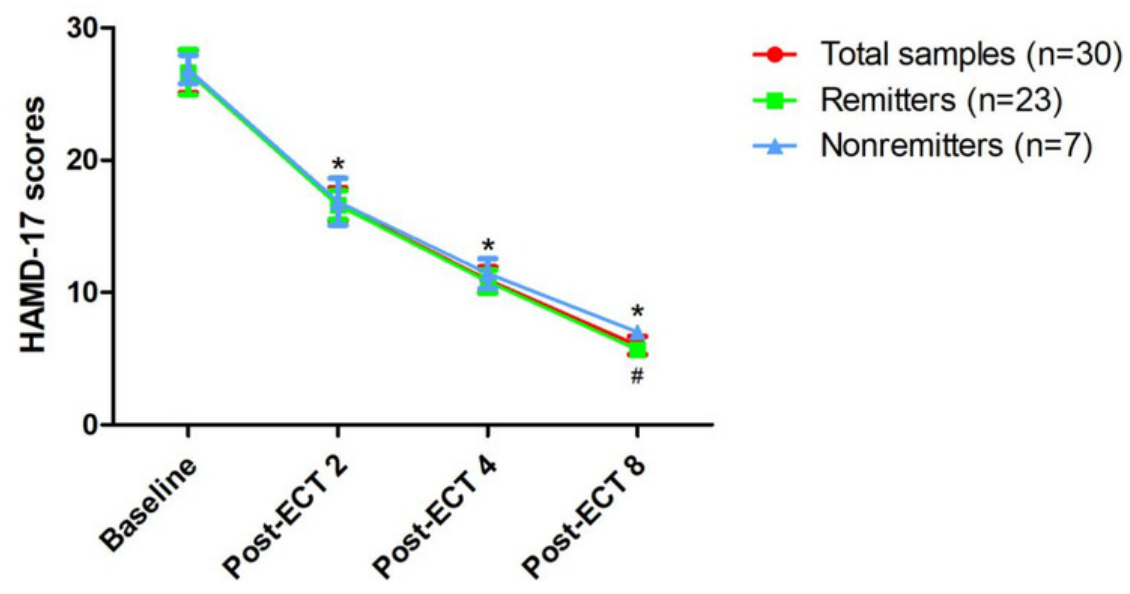

"Significant difference was found at any of the indicated times when compared baseline $(p<0.05)$. \#Significant difference was found between remitters and nonremitters at indicated times $(p<0.05)$. Abbreviations: ECT=electroconvulsive therapy; HAMD-17=the 17-item Hamilton Depression Rating Scale. 


\section{Figure 2}

Changes in serum BDNF levels following eight ECT treatments

Notes: no significant difference was found at any of the indicated times when compared to baseline across the total sample, even among remitters and nonremitters ( $p>0.05$ ); no significant difference was found between remitters and nonremitters at any of the indicated times $(p>0.05)$. Abbreviations: $B D N F=$ brain-derived neurotrophic factor; $\mathrm{ECT}=$ electroconvulsive therapy; $\mathrm{TRD}=$ treatment-refractory depression. 


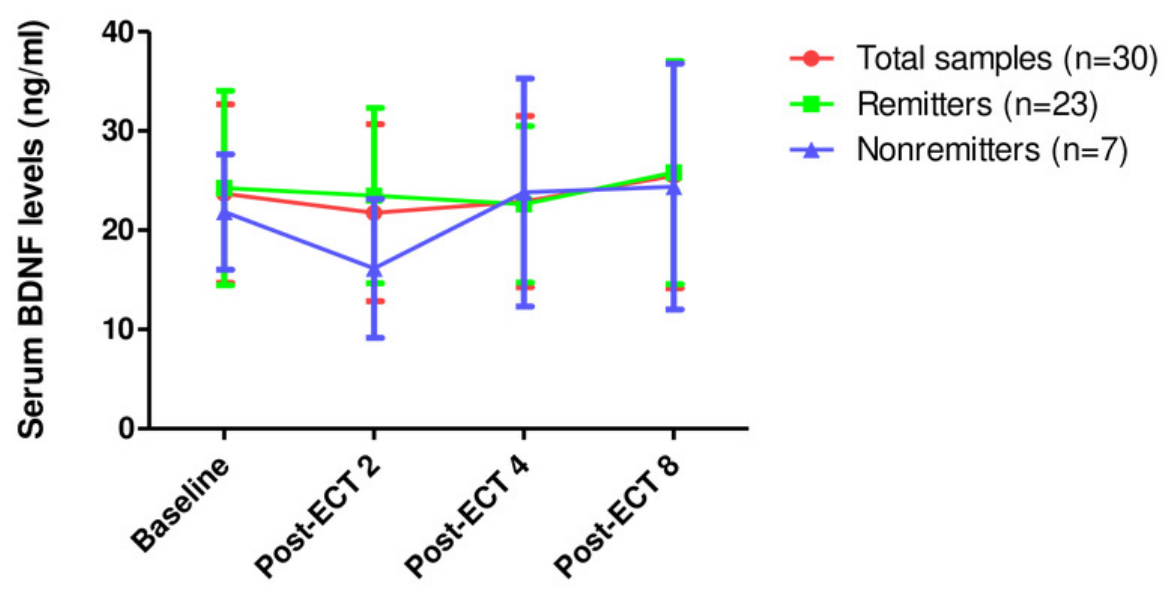

Notes: no significant difference was found at any of the indicated times when compared to baseline across the total sample, even among remitters and nonremitters ( $p>0.05)$; no significant difference was found between remitters and nonremitters at any of the indicated times $(p>0.05)$.

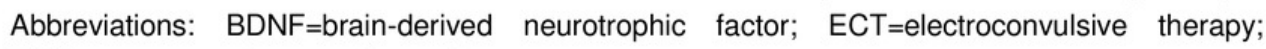
$\mathrm{TRD}=$ treatment-refractory depression. 